\title{
ANALISIS DEL PROBLEMA DE INTRUSION MARINA DEL ACUIFERO DE CHILCA MEDIANTE MODELAMIENTO NUMERICO
}

\author{
Douglas D. Sarango J. ${ }^{\mathrm{a}}$ \\ ${ }^{a}$ Departamento de Ingeniería Mecánica de Fluidos, Facultad de Ciencias Físicas \\ Universidad Nacional Mayor de San Marcos, Apartado Postal 14-0149, Lima, Perú
}

\begin{abstract}
Resumen
La presente investigación tiene como objetivo el análisis y la evaluación del problema actual de intrusión marina del acuífero de Chilca mediante la modelación númerica. Para cumplir con este objetivo es necesario contar con un manejo apropiado de este sistema acuífero y establecer politicas de operación, manejo y conservación, para lo cual se debe efectuar un estudio del comportamiento espacial y temporal de este recurso hídrico. En este sentido, se ha efectuado el modelamiento númerico del acuífero de Chilca en regimen permanente mediante la solución de las ecuaciones que gobiernan el flujo del agua subterránea en medios porosos, utilizandose para ello la técnica de elementos finitos con el uso de mallas triangulares desarrollado en el Modelo Micro-Fem V.3. Se genero 143 elementos triangulares y 100 nodos, asignando a cada elemento datos de piezometria, caudal de explotación, trasmisibilidad, etc. Con el modelo númerico calibrado se simulo las hipotesis de explotación futura plantedas por el sector agricultura, determinandose de esta forma la nueva posición del nivel del agua subterránea en el área del acuífero de Chilca. La ubicación de la interfase agua dulce - agua salada, que representa el problema de intrusión marina, fue determinada aplicando la condición de Ghyben-Herzberg a los resultados de los nuevos niveles del agua subterránea obtenidos al simular las hipotesis de explotación plantedas. Los resultados indican que en las zonas del valle de Chilca denominadas S2, S3, S4, S5 y S6 los pozos ubicados en ellas extraerian agua contaminada con alto contenido de cloruro de sodio. Mientras que la zona S1, solo podria ser explotada vía pozos para poder abastecer de agua potable a la ciudad de Chilca, ubicada a mitad del valle.
\end{abstract}

Palabras claves: Acuífero Chilca, intrusión marina, modelo numérico, método de elementos finitos.

\begin{abstract}
The project has as objectives the analysis and evaluation of the nowadays problem given by the marine intrusion in the Chilca aquife, this will be done through numeric modeling. of the aquifer. In order to fulfill these objectives it is necessary to count with an appropriate handling of this aquifer system. Also it will be necessity to establish policies of operation, handling and conservation. In order to do so, a study of the spatial and temporal should be affected on this water resource. The numeric modeling of the Chilca aquifer has been affected under permanent regimen through the solution of the underground water flux equations for porous environment. The finite elements technique with the use of triangular nets was used, this was developed on the Micro Fem V.3 model. 143 triangular nets and 100 nodes were generated, given to each element data of water level, exploitation flow, transmissivities, etc. With the numeric calibrated model, the future exploitation hypothesis were simulated which were given by the agriculture sector. Through this, it was determinated the way for the new underwater level position on the area of Chilca aquifer. The sweet water / salt water interface location represents the marine intrusion problem. It was determinated applying the Ghyben-Herzberg conditions to the results from the new underground water levels obtained by la simulating the given exploitation hypothesis. The results indicate that the zones denominated S2, S3, S4, S5 and S6 on the valley of Chilca, the wells locate on the mentioned zones shall extract contaminated water with high sodium chloride content. Meanwhile, the S1 zone could only be exploited through wells in order to supply potable water to the city of Chilca located in the middle of the valley.
\end{abstract}

Keywords: Chilca aquifer, marine intrusion, numerical model, finite elements method. 


\section{Introducción}

La sobre explotación del acuífero de Chilca para el abastecimiento de la demanda de agua de uso domestico y agrícola ha producido en los últimos años un incremento de la salinidad del agua extraída, como consecuencia de la intrusión marina en el acuífero [1],[2]. Hecho que ha sido comprobado por las evaluaciones que viene efectuando la Dirección General de Aguas y Suelos del INRENA. Muestreos efectuados entre los meses de Mayo a Junio de 1982, en un total de 63 muestras de agua de 59 pozos, 2 de mar y 2 de afloramientos, han dado valores de conductividades eléctricas entre $0.70 \mathrm{mmhos} / \mathrm{cm}$ y $17.2 \mathrm{mmhos} / \mathrm{cm}$ a $25^{\circ} \mathrm{C}$, indicando alta concentración salina localizada al Noroeste del poblado de Chilca, así como al sur del valle, zona denominada Las Salinas. La evolución de la concentración salina desde el año 1966 a 1997 en las aguas del acuífero de Chilca indica un ascenso fuerte de la concentración salina a través de los años, incrementándose entre 4 a 8 veces la concentración inicial. Existe interés de parte del sector de agricultura en seguir explotando el acuífero mediante la implementación de dos nuevos proyectos de irrigación, que implica en la perforación de 172 nuevos pozos de agua subterránea, el primer proyecto con un caudal de explotación de 2000 1/s mediante 86 pozos localizados en los 6 sectores de valle de Chilca, $\mathrm{y}$ el segundo proyecto igual al primero pero con un caudal de 1000 1/s. Dada la problemática actual de intrusión marina en el acuífero y siendo necesario su análisis y control dentro del acuífero, se simulo su explotación considerando las dos implementaciones y de esta forma determinar la evolución de la interfase agua dulce-agua salada a aplicando el modelo MicroFem V.3 que es un modelo de elementos finitos, [3]. Con los resultados de estas simulaciones se determinaron los planos de los niveles de intrusión marina bajo condiciones de GhybenHerzberg, [2].

\section{Planteamiento de Hipótesis}

Incremento del problema de intrusión marina en el acuífero de Chilca por la implementación de dos nuevos proyectos de irrigación cuya demanda de agua se dará mediante la perforación de nuevos pozos de explotación .

\section{Materiales y Métodos}

Los modelos matemáticos son resultado de la aplicación de los principios físicos de conservación que através de ecuaciones intentan explicar el movimiento del agua subterránea en el contexto de las particularidades geométricas, hidrogeológicas, hidrológicas, condiciones de frontera y de explotación que caracterizan al acuífero a modelar, [4], [5]. Al no existir una solución analítica general para la solución de la ecuación que gobierna el flujo de agua subterránea en un acuífero, los modelos matemáticos se basan en ecuaciones matemáticas continuas que son solucionadas con técnicas numéricas de aproximación. Su expresión se hace particular con la introducción de las restricciones impuestas por la realidad física del acuífero y los criterios utilizados. Dentro de esta concepción el modelo matemático de un acuífero en general tiene los siguientes pasos: (a) El modelo requiere información sobre la geometría del acuífero y sus propiedades hidrogeológicas, (b) El modelo opera sobre un estado inicial conocido (condiciones iniciales y de contorno) que se expresa en forma de un mapa de hidroisohipsas correspondientes a la fecha (datos observados), (c) El modelo requiere información sobre los procesos de recarga y descarga que ocurren a partir de esa fecha y mediante un periodo determinado de tiempo, (d) El modelo calcula el estado del acuífero al final de este periodo, resultado de la combinación de los datos expresados en los pasos a), b), c) y se expresan en mapas de hidroisohipsas correspondiente a la fecha final del periodo de tiempo seleccionado, y (e ) Cualquier variación de los datos en los pasos a), b), y c) se refleja en el pasos d), [6], [7]. En este sentido, si se simula situaciones históricas, las respuestas del modelo deben coincidir con los estados previamente conocidos en el acuífero, comprobando hasta que punto el modelo puede ser confiable y ser capaz de reproducir el comportamiento real.

3.1 Modelamiento del Flujo de Aguas Subterráneas mediante el Método de Elementos Finitos - Modelo MICRO-FEM V.3

El método de elementos finitos es una técnica para el cálculo de las cargas en diferentes puntos 
del acuífero en el tiempo y en el espacio del sistema acuífero, [3]. Partiendo primeramente de las ecuaciones de Laplace para flujo permanente y flujo transitorio respectivamente, se tiene:

$$
\nabla^{2} \phi=\frac{S}{T} \frac{\partial \phi}{\partial t}
$$

El primer paso es establecer la solución de prueba

$$
\mathrm{H}_{(\mathrm{x}, \mathrm{y})}=\sum_{i=1}^{N} N_{i(x, y)} h_{i}
$$

Donde:

$\begin{array}{ll}\mathrm{H}_{(x, y)} & =\text { Solución de prueba } \\ \mathrm{N}_{\mathrm{i}(x, y)} & =\text { Factor de interpolación. } \\ \mathrm{h}_{\mathrm{i}} & =\text { Carga en el nodo } \mathrm{L} \\ \mathrm{N}_{\mathrm{i}} & =\text { Número de nodos en el sistema }\end{array}$ acuífero.

$$
\iint\left(\frac{\partial^{2} h}{\partial x^{2}}+\frac{\partial^{2} h}{\partial y^{2}}\right) N L_{(x, y)} \partial x \partial y=0
$$

La ecuación de continuidad para el flujo de aguas subterráneas en un acuífero confinado, anisotrópico, tridimensional en régimen transitorio puede ser expresada como:

$\frac{\partial}{\partial x}\left(K_{x x} \frac{\partial h}{\partial x}\right)+\frac{\partial}{\partial y}\left(K_{y y} \frac{\partial h}{\partial y}\right)+\frac{\partial}{\partial z}\left(K_{z z} \frac{\partial h}{\partial z}\right)+q-S \frac{\partial h}{\partial t}=0$

Donde:

$\mathrm{h}$ : Carga hidráulica total(L)

$\mathrm{K}_{\mathrm{xx}}, \mathrm{K}_{\mathrm{yy}}, \mathrm{K}_{\mathrm{zz}}$ : Conductividad hidráulicas (L/T)

$\mathrm{q}$ : Caudal de recarga y descarga $\left(\mathrm{L}^{3} / \mathrm{T}\right)$

$\mathrm{S}$ : Almacenamiento especifico del material poroso

$\mathrm{t}:$ Tiempo $(\mathrm{t})$

En general $S, K_{x x}, K_{y y}, K_{z z}$ pueden ser funciones del espacio, es decir, $S=S_{(x, y, z)}$ y $K=K_{(x, y, z)}$; h y q pueden ser funciones del espacio y el tiempo, $\mathrm{h}=\mathrm{h}_{(\mathrm{x}, \mathrm{y}, \mathrm{z}, \mathrm{t})}$ y $\mathrm{q}=\mathrm{q}_{(\mathrm{x}, \mathrm{y}, \mathrm{z}, \mathrm{t})}$ de este modo la ecuación anterior describe el flujo de agua subterránea bajo condiciones de equilibrio en un medio heterogéneo y anisotrópico. La ecuación anterior conjuntamente con las especificaciones del flujo y/o condiciones de frontera del sistema acuífero y la condición inicial constituye la ecuación diferencial de flujo de agua subterránea. En un medio acuífero. La técnica de elementos finitos involucra resolver la ecuación diferencial de flujo del agua subterránea por medio del calculo variacional. Para obtener la solución numérica de la ecuación anterior, el acuífero es subdividido en elementos finitos.

$$
\left.F=\iint\left[\frac{K x x}{2}\left(\frac{\partial h}{\partial x}\right)^{2}+\frac{K y y}{2}\left(\frac{\partial h}{\partial y}\right)^{2}+\frac{K z z}{2}\left(\frac{\partial h}{\partial z}\right)^{2}+\left(S \frac{\partial h}{\partial t}-q\right)\right]\right\rfloor \partial x \partial y \partial z
$$

El tamaño y la forma de los elementos finitos son arbitrarios y generalmente es triangular, siendo más pequeños en lugares en los cuales se requiere mayor precisión. Los parámetros $\mathrm{K}_{\mathrm{xx}}$, $\mathrm{K}_{\mathrm{yy}}, \mathrm{K}_{\mathrm{zz}}, \mathrm{S}, \mathrm{q}$ son conservados constantes para un elemento dado, pero varían de elemento en elemento. Para minimizar la ecuación diferencial $\partial \mathrm{F} / \partial \mathrm{h}$ es evaluada para cada nodo e igualada a cero, el sistema de ecuaciones simultáneas resultante puede ser resuelto rápidamente mediante el modelo Micro-Fem V.3. El modelo Micro-Fem V.3 (Finite Element Method Versión 3 ), utiliza métodos numéricos con elementos finitos usando mallas triangulares para solucionar las ecuaciones de flujo de agua subterránea (acuífero libre, confinado y semiconfinado) en régimen permanente y no permanente, y esta constituido por los siguientes subprogramas:

\section{FemGrid : $\quad$ Genera mallas triangulares}

FeModel: $\quad$ Modifica el mallado, define los parámetros, caudales y condiciones de frontera del acuífero y proporciona una presentación gráfica de los datos ingresados, con la finalidad de analizar e interpretar los resultados del Modelo.

FemCalc: Calcula las cargas en los nodos FemPlot : $\quad$ Plotea el mallado, la distribución de las cargas, el acuífero y sus propiedades

FeMerge: Compila un nuevo modelo basado en un modelo existentes y nuevos datos del mallado

De otro lado, la ubicación de la interfase agua dulce-agua salada, que representa el problema de intrusión marina, se determina aplicando la condición de Ghyben-Herzberg a los resultados de los nuevos niveles de agua subterránea obtenidos en el acuífero al simular las hipótesis de explotación planteadas, la posición de esta interfase se determina por la relación siguiente: 
dónde:

$$
\mathrm{H}_{\mathrm{s}}=40 \mathrm{H}_{\mathrm{f}}
$$

$\mathrm{H}_{\mathrm{s}}=$ Profundidad a la cual se halla la interfase agua dulce-agua salada

$\mathrm{H}_{\mathrm{f}}=$ Nivel del agua subterránea

$40=$ Valor deducido de las densidades de agua dulce y agua salada (Ghyben-Herzberg).

\subsection{Información Utilizada}

El Acuífero del Valle de Chilca, está ubicado en el departamento de Lima, entre los paralelos $12^{0} 28^{\prime}$ y $12^{0} 39^{\prime}$ de Latitud Sur y los meridianos $76^{\circ} 40^{\prime}$ y $76^{\circ} 45^{\prime}$ de Longitud Oeste; limitando por el Norte con la cuenca del Río Lurín; por el Este con la quebrada del río de Chilca y por el Sur Este con el Océano Pacifico. El área del acuífero es aproximadamente $58 \mathrm{~km}^{2}$. Se eleva desde el nivel del mar hasta los $100 \mathrm{msnm}$, siendo el mayor ancho de Este a Oeste de 8,75 $\mathrm{Km}$. y el largo de Norte a Sur de $9,5 \mathrm{Km}$. La información utilizada en la etapa de calibración del modelo Micro-Fem V.3 para el acuífero de Chilca es la siguiente: (a) Delimitación Espacial del Acuífero, (b) Topografía, (c) Bordes Impermeables, (d) Potenciales Impuestos, (e) Extracción por bombeo, (f) Estado inicial del Acuífero, (g) Propiedades Hidrogeológicas, (h) Recarga del Acuífero y (i) Infiltraciones por riego. En la etapa de simulación, la información de entrada son los resultados de la etapa de calibración y los caudales de explotación de las Hipótesis No. 1 y No.2 planteadas para la explotación futura del acuífero de Chilca. Para la mejor presentación y análisis de los resultados estos han sido procesados con el modelo Surfer For Windows V.3.

\subsection{Metodología}

El modelamiento del acuífero de Chilca se efectúo en dos etapas: Etapa de calibración y Etapa de simulación.

\subsubsection{Etapa de Calibración}

En esta etapa se busca reproducir mapas de hidroisohipsas en base a niveles de agua observados históricamente, considerados como estado final del acuífero. El procedimiento seguido para ejecutar esta etapa es el siguiente, [4], [6]: a) Recopilación y análisis de la información.

b) Selección del periodo de calibración

c) Límites del acuífero

d) Discretización espacial del acuífero

e) Configuración de la malla

f) Determinación de las propiedades hidrogeológicas

g) Flujos impuestos

h) Estado inicial del acuífero

i) Condiciones de frontera

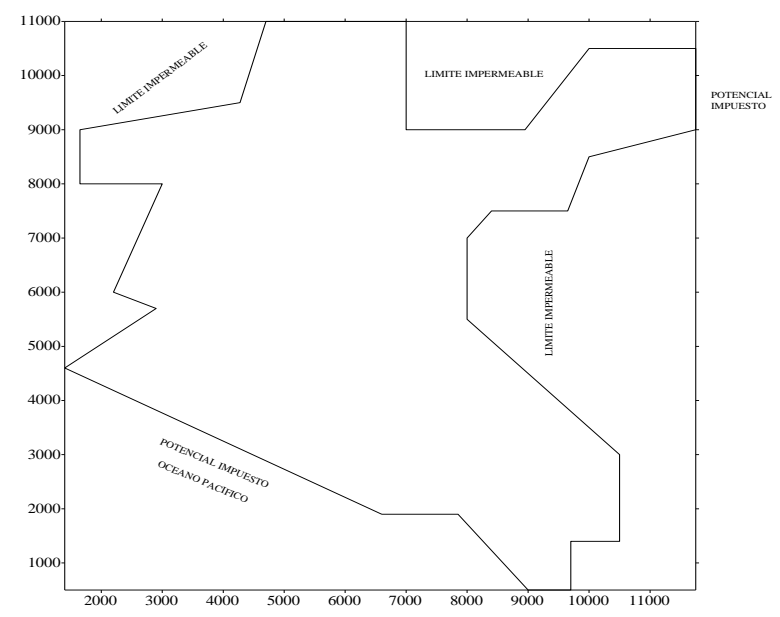

Fig. 3.1 Discretización del acuífero de Chilca.

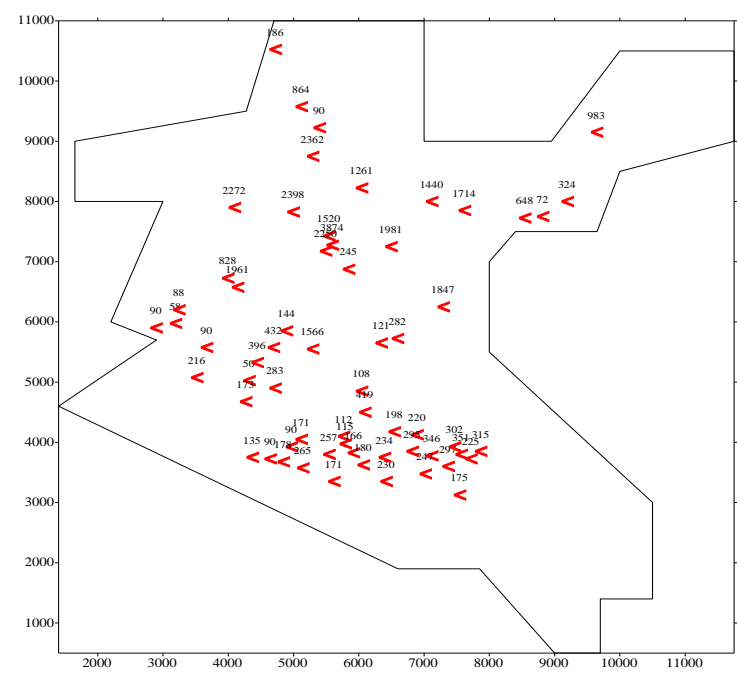

Fig. 3.2 Ubicación de pozos de explotación

\subsubsection{Etapa de Simulación}

Una vez obtenida la confiabilidad del modelo en la etapa de calibración, este se utilizó para obtener los estados finales del acuífero sometido a los regímenes de explotación siguiente, [4], 
[6]: (a) Hipótesis de explotación No. 1: El acuífero será sometido a una explotación de $2,000 \mathrm{lt} / \mathrm{s}$ (86 pozos), incremento de $501 \mathrm{lt} / \mathrm{s}$ respecto al estado inicial de explotación, con lo cual se cubre la demanda de agua de los cultivos en un área de 1,660 Ha., que constituye el 75\% de las tierras cultivadas en el valle de Chilca, y (b) Hipótesis de explotación No. 2:Explotación del acuífero en $1,000 \mathrm{lt} / \mathrm{s}$ (86 pozos), disminuyendo en $506 \mathrm{lt} / \mathrm{s}$ respecto al estado inicial de explotación, con lo cual se pretende controlar el avance de la intrusión marina en el acuífero de Chilca.

\section{Resultados y Discusión}

\subsection{Resultados de la Etapa de Calibración}

La calibración del modelo Micro-Fem V.3 para la simulación del Acuífero de Chilca se realizó bajo la condición de régimen permanente, para lo cual se simuló el comportamiento de la napa para el mes de Junio de 1982, período donde de cuenta con información de buena calidad. Se genero 143 mallas triangulares y 100 nodos, ver Figura 4.1. Se asignaron valores de trasmisibilidad y piezometría a todos los nodos de este mallado triangular por superposición del plano de trasmisibilidad, y del plano de isoprofundidad respectivo. Los caudales de explotación para cada uno de los nodos del mallado triangular, se obtuvieron superponiendo al mallado el plano de ubicación de los pozos con sus respectivos caudales de explotación. Los caudales de recarga por infiltración del acuífero fueron asignados a los nodos 14, 37 y 13 . Siendo la recarga total por infiltración de 23,468 $\mathrm{m}^{3} / \mathrm{d}$, se asigno un $25 \%$ a los nodos 14 y 13 y en $50 \%$ al nodo 37 , es decir valores de $5,867 \mathrm{~m}^{3} /$ día y $11,734 \mathrm{~m}^{3} /$ día respectivamente $\mathrm{El}$ proceso de calibración será aceptado si la piezometría asignada ó observada y la piezometría calculada por el modelo tengan valores iguales y/o semejantes. Al inicio del proceso de calibración estos valores fueron diferentes lo cual obligo a efectuar cambios en los valores de transmisivilidad y piezometría asignada inicialmente, que han sido del orden del 5\% al $10 \%$. Una forma de poder visualizar el proceso de calibración es mediante el ploteo de las curvas de piezometría asignada y la calculada teniendo como base la delimitación espacial del acuífero. Una cercanía, y una misma tendencia de estas curvas indican la aceptación o rechazo del proceso de calibración efectuado. En la Figura 4.2 se muestra el resultado final del proceso de calibración efectuado para el acuífero de Chilca, observándose que el mayor ajustes de las curvas piezométricas asignadas y las calculadas están localizadas en la quebrada del Valle y el la zona de mayor explotación del acuífero. Las mayores diferencias se localizan en la zona cercana al litoral, zona en la cual no se contó con información de isoprofundidad y trasmisibilidad.

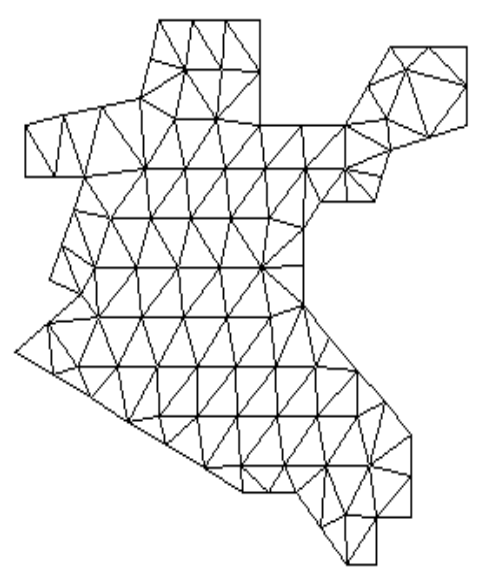

Fig. 4.1 Mallado triangular del acuífero de Chilca

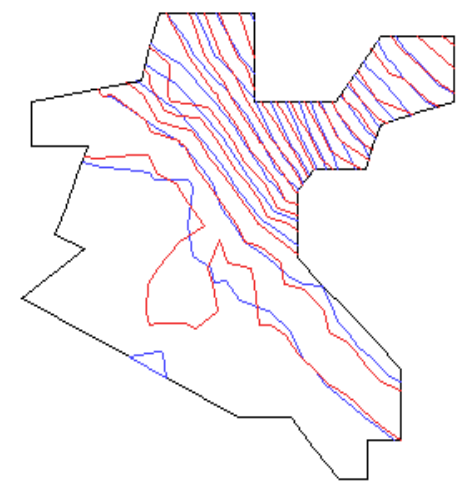

Fig. 4.2 Proceso de Calibración del acuífero de Chilca

\subsection{Resultados de la Etapa de Simulación}

Una vez calibrado el modelo del Acuífero de Chilca, este fue simulado bajo las hipótesis de explotación indicadas en el ítem 3.2.2., se obtuvieron los nuevos niveles de agua subterránea en el área de estudio del acuífero. 


\section{Hipótesis de explotación No. 1}

Para el análisis de los resultados de la simulación de esta hipótesis, se han superpuesto los resultados de niveles de aguas subterránea simulados y los obtenidos en la etapa de calibración del acuífero de Chilca, tal como se muestra en la Figura 4.3, donde se aprecia que en el sector $\mathrm{S} 1$ el nivel del agua a descendido entre 0.5 y $1.0 \mathrm{~m}$. respecto al estado inicial del acuífero (etapa de calibración), y $0.5 \mathrm{~m}$. en el sector S2 y S4.

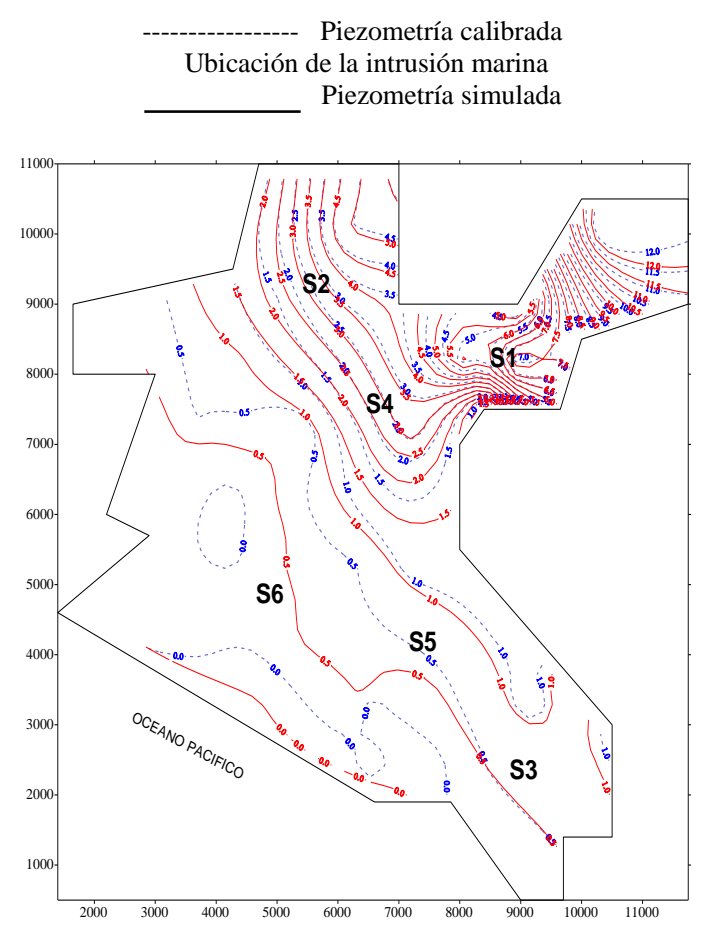

Fig. 4.3 Posición de napa freática en el acuífero de Chilca - Hipótesis No. 1

En la Figura 4.4, se presentan los resultados de la ubicación de la intrusión marina al aplicar la relación de Ghyben-Herzberg a los niveles de agua subterránea obtenida al simular la Hipótesis de Explotación No. 1. La intrusión marina se extiende desde la zona $\mathrm{S} 1$ a un nivel de -150 msnm, pasando por el pueblo de Chilca (parte central del valle) a $-25 \mathrm{msnm}$, hasta la línea de playa a $-10 \mathrm{msnm}$. Considerando que el promedio de profundidad de los pozos tubulares es de 25 y $35 \mathrm{~m}$. y la profundidad media de los pozos a tajo abierto son menores a $10 \mathrm{~m}$., y menos de $10 \mathrm{~m}$. de los pozos a tajo abierto, estos estarán contaminados al atravesar la interfase agua dulce-agua salada en los sectores S3, S5 y
S6, y parte en la zona S4. Los pozos de las zonas S1 y S2 no presentarán ninguna contaminación salina en vista que la interfase agua dulce-agua salada se encuentra a más de $-100 \mathrm{msnm}$ sobre el nivel del mar.

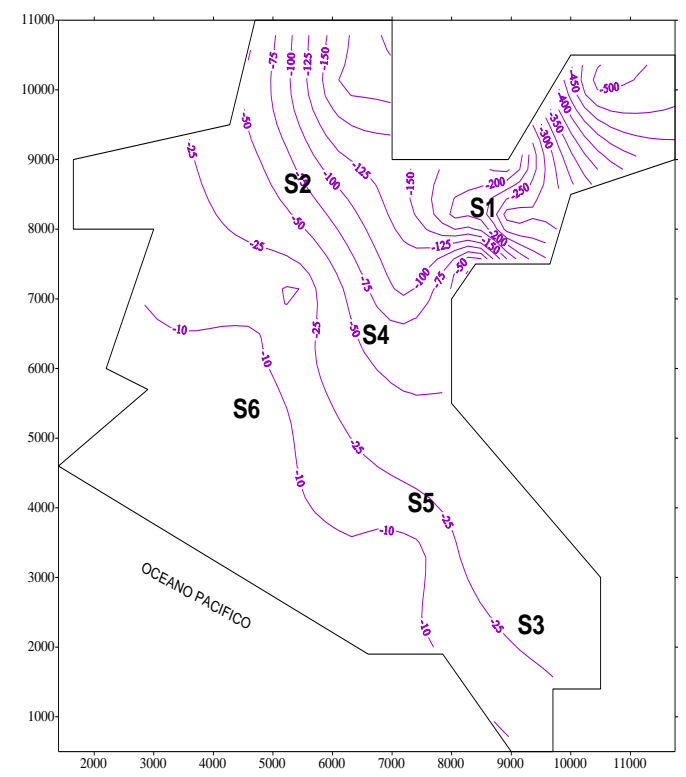

Fig. 4.4 Ubicación de la intrusión marina en el acuífero de Chilca - Hipótesis No. 1

\section{Hipótesis de explotación No. 2}

El acuífero ha sido simulado con explotación de $1,000 \mathrm{lt} / \mathrm{s}$, disminuyendo en $506 \mathrm{lt} / \mathrm{s}$ respecto al estado inicial de explotación (etapa de Calibración). Los resultados presentados en la Figura 4.5, indican que en los sectores $\mathrm{S} 1, \mathrm{~S} 2$ y $\mathrm{S} 4$ el nivel del agua a descendido $0.5 \mathrm{~m}$. respecto al estado inicial del acuífero, y en los sectores S3, S5, S6 y S4 ha sufrido una ligera variación con respecto al nivel del estado inicial, por lo tanto sigue habiendo una sobre explotación del acuífero de Chilca. Respecto a la intrusión marina en la Figura 4.6, se aprecia que esta se extiende desde la zona S1 a un nivel de -150 msnm, pasando por el pueblo de Chilca a -50 msnm, hasta la línea de playa a $-10 \mathrm{msnm}$, bajo estas condiciones y considerando la profundidad de los pozos tubulares y a tajo abierto, en las zonas S3, S4, S5 y S6, estos atravesarán la interfase aguas dulce-agua salada, extrayendo agua con alto contenido de cloruro de sodio. Los pozos de las zonas S1 y S2 no presentarán ninguna contaminación salina en vista que la 
interfase agua dulce-agua salada se encuentra a mas de -150 msnm sobre el nivel del mar.

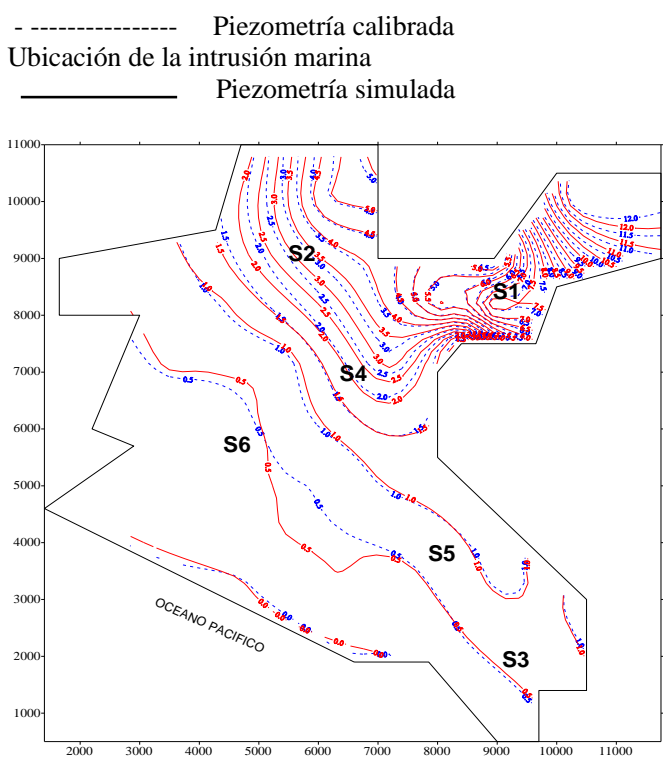

Fig. 4.5 Posición de napa freática en el acuífero de Chilca Hipótesis No. 2

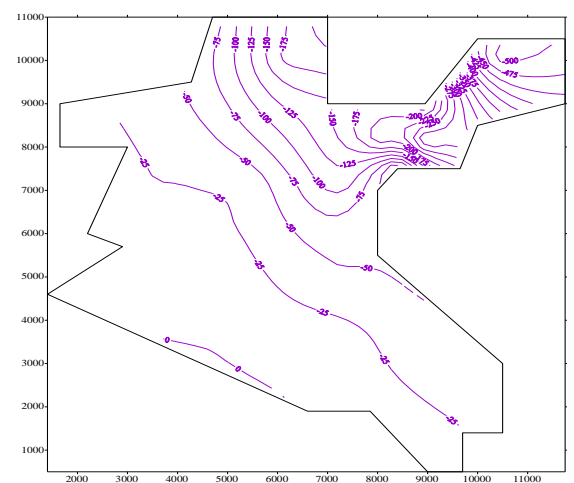

Fig. 4.6 Posición de napa freática en el acuífero de Chilca Hipótesis No. 2

\section{Conclusiones y Recomendaciones}

El modelo MICRO - FEM V.3 aplicado al Acuífero de Chilca, utiliza métodos numéricos con elementos finitos usando mallas triangulares para solucionar las ecuaciones de flujo de agua subterránea (acuífero libre, confirmado y semiconfinado) en régimen permanente y no permanente. En la etapa de calibración del modelo del Acuífero de Chilca se logró reproducir su comportamiento para el mes de Junio de 1982. De implementarse la Hipótesis de Explotación No. 1, de 2,000lt/s para cubrir las necesidades de agua de 1,660 $\mathrm{Ha}$ del valle de Chilca, la interfase agua dulce-agua salada se ubica a $-25 \mathrm{msnm}$ en los sectores $\mathrm{S} 3, \mathrm{~S} 4$ y S3 del valle de Chilca, y a $-10 \mathrm{msnm}$ en el sector S6. Todo el acuífero de Chilca esta contaminado excepto el sector S1 donde la intrusión marina se ubica en promedio a $-200 \mathrm{msnm}$. Existe por lo tanto una sobreexplotación del acuífero que supera la reserva reguladora del acuífero, que es de 348 lt/s. Los resultados de la simulación de la Hipótesis de Explotación No. 2 de 1,000lt/s en el acuífero de Chilca, indican que existe intrusión marina en los sectores S3, S4, S5 y S6, cuyos pozos atravesarán la interfase agua dulceagua salada. Los pozos de las zonas S1 y S2 no presentarán problemas de intrusión marina en vista que la interfase agua dulce - agua salada esta se encuentra a más de -100 msnm. Declarar al acuífero de Chilca en emergencia y evitar toda explotación por medio de pozos en los sectores S2, S3, S4, S5 y S6. El sector S1 debe de ser explotado solo para cubrir las demandas de uso poblacional de la ciudad de Chilca.

Estudiar la posibilidad de efectuar una recarga artificial del acuífero de Chilca.

\section{Referencias}

[1] Abbott, M.B. Computational Hydraulics, Elements of the Theory of Free Surface Flows, Pitman Publishing Limited, London (1985).

[2] Custodio E., Lamas M. Hidrologia Subterránea. Tomo II. Ediciones Omega S.A., Barcelona Espana (1983)

[3] Waterloo Hydrogeologic Software. User Manual MicroFem V.3., Canada (1992).

[4] Amisial, R., Jegat H. Aprovechamiento y Modelos de Aguas Subterráneas, CIDIAT, Merida Venezuela (1976)

[5] Bear, J., Verruijt A. Modelling Groundwater Flow and Pollution. D. Reidel Publishing Company. Dordrecht Holanda (1987).

[6] Sarango D. Modelo Matemático en Regimen Transitorio para el Control de la Intrusión Marina para el Acuifero de la Yarada y Hospicio. Tacna-Perú. Tesis Profesional EAPIMF, UNMSM, Lima (1982).

[7] Jegat H. Evaluación estocastica de politicas de explotación de agua subterránea en el valle de Quibor. Anales cientificos, UNALM, Lima (2003). 